polymer directly with the appropriate amine or sodium alkoxide.

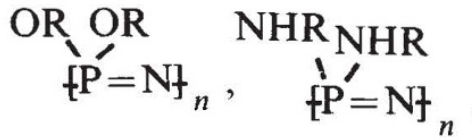

$$
\begin{gathered}
\mathrm{NR}_{2} \mathrm{NR}_{2} \\
1 / 1 \\
\mathrm{PP}=\mathrm{N} f_{n}
\end{gathered}
$$

Since then Allcock's group at Pennsylvania State University have published about 30 papers describing the synthesis and properties of a large number of these polymers (see Science 193, 1214, 1976; and Chemtech 553, September 1975). The interest of these polymers lies in this versatility, an indefinite number of side groups can easily be added to produce a wide range of properties without having to perform a new polymerisation each time. The low temperature flexibility of some of these materials can also be applied.

Low temperature elasticity is necessary not only to make rubber grommets for use in cold climates but also in the pumping and handling of cold liquids. A necessary condition for this is believed to be chain flexibility, that is, unhindered rotation about the chain backbone bonds. In the polyphosphazenes and silicone rubbers this arises by the alteration of an unsubstituted chain atom (the nitrogen) with one carrying only small substitutes (the phosphorus) so that internal rotation can occur without the side groups colliding. Allcock, Allen and Meister (Macromolecules 9, 950, 956; 1976) have made a start toward quantifying this by calculating the potential energy as a function of conformation for a series of halo- and organo-substituted polyphosphazenes. These calculations assume a Lennard-Jones interatomic potential and then evaluate the potential energy due to neighbouring groups on the chain as a function of the bond angles. However the chain does have a conjugated double bond structure which might also hinder rotation, although Allcock asserts that it does not on the basis that the orbitals will be symmetric about the bond axis. The exact nature of the P-N double bond is still a matter for debate and they may have to be taken into account (Allcock Phosphorus-Nitrogen Compounds Academic, London-New York, 1972). The potential energy barrier to bond rotation calculated in this way is directly related to the chain flexibility and can be compared with the polymer glass transition temperature which varies from $180 \mathrm{~K}$ for the difluoro polymer to $265 \mathrm{~K}$ for poly(diphenoxyphosphazene). The calculated barrier heights correlate

\title{
Ultraviolet windows in sunglasses
}

\author{
from John Walker
}

$C_{\text {AN }}$ sunglasses damage the eyes? Two American scientists have discovered that many sunglasses on sale in the United States are not effective in filtering out ultraviolet radiation; they may actually increase the possibility of eye damage in bright sunlight.

Visible light has wavelengths between $400 \mathrm{~nm}$ (blue/violet) and $700 \mathrm{~nm}$ (Fig. 1). Other wavelengths, which are at best unnecessary for vision and at worst a danger to the eyes, ought to be removed by sunglasses before reaching the eyes. Ultraviolet $(300-400 \mathrm{~nm})$ in particular is suspected of causing cataracts.

Anderson and Gebel (Appl. Optics $16,515 ; 1977)$ tested sunglasses they had randomly purchased in local shops and drugstores (in Ohio, USA). Many of them were optically unsatisfactory and " . . . seem to be designed more to satisfy the fashion whims of the wearer than to shield the eyes from unnecessary radiation". One-third of their selection transmitted more light in the ultraviolet than in the visible-in the worst case $70 \%$ transmission at $360 \mathrm{~nm}$ compared with $20 \%$ at $550 \mathrm{~nm}$ (Fig. 1). This may be dangerous for two reasons.

First, as the amount of visible light reaching the eye is cut down, much stronger outside light levels can be tolerated without discomfort, and so the ultraviolet exposure may be greater than if no sunglasses were worn. Alternatively, the reduced visible light intensity causes the pupil to dilate allowing more light to enter the eye. Anderson and Gebel calculate that the worst sunglasses

well with the observed glass transition temperatures for the halo- and organosubstituted polymers separately but poorly when the two groups are compared, the organo compounds having larger barriers to rotation for similar glass transition temperatures. Clearly some other factor must be taken into account.

This question should really be taken further as there has been relatively little advance since the theory of Gibbs and DiMarzio in 1962 stressed the importance of chain flexibility for the glass transition. The polyphosphazenes provide a good system on which to relate properly the calculated conformational energies to the glass transition temperatures since the ease with which substituents can be changed on the same backbone should allow the isolation of factors such as polarity, actually double the amount of ultraviolet absorbed by the eye (Fig. 1); thus there is a greater risk of eye damage than if no sunglasses were worn.

Some sunglasses were quite satisfactory, however. Figure 1 shows the transmission spectrum of one of the better pairs-though even there it would be better if the transmission cut off at $450 \mathrm{~nm}$ instead of $390 \mathrm{~nm}$. And unfortunately there appeared to be no correlation between ultraviolet performance, price, lens colour or manufacturer; there is no way of picking a good pair other than by measuring its transmission spectrum.

The authors conclude that their preliminary study should be extended, and should include prescription sunglasses (the only pair they tested performed very well). Furthermore "... it would be in the consumers' interest for manufacturers to indicate in some manner the transmission characteristics of their lenses over the solar spectrum".

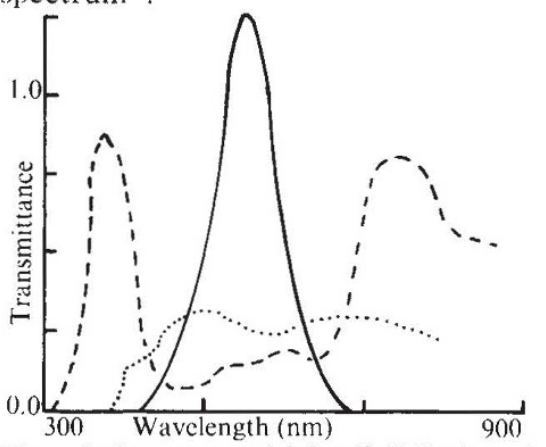

The relative eye sensitivity (full line), and optical transmission curves for the worst (dashes) and best (dots) pairs of sunglasses. side group flexibility and copolymer composition.

The proposed uses for the polyphosphazenes also reflect the effect of side groups on the polymer properties. Small side groups give a low glass transition temperature and resistance to swelling by hydrocarbons, desirable for gaskets and O-rings. Crystallisation is prevented by the use of mixed substituents so that the polymer is rubbery. With some subtlety it should be possible to produce a rubber which crystallises only on stretching. This would give good strength properties comparable to natural rubber rather than to silicone rubber which cannot crystallise and is weak. By adding fluorinated substituents polymers can be produced with a low surface energy which could be used for water repellent coatings and for surgical implants 